\title{
Adaptación de dos cuestionarios de motivación: Autorregulación del Aprendizaje y Clima de Aprendizaje
}

\author{
Lennia Matos Fernández
}

Universidad de Lima (Perú)

Recibido: 11 de mayo del 2009 / Aprobado: 24 de agosto del 2009

Esta investigación psicométrica tuvo como objetivo adaptar y validar dos cuestionarios: Autorregulación del Aprendizaje y Clima de Aprendizaje. La muestra estuvo compuesta por 369 estudiantes universitarios de una universidad privada de Lima con una edad promedio de 19.30 años $(D E=2.49)$. En ambos casos, se analizó la validez de constructo a través de análisis factoriales exploratorios con extracción de componentes principales, análisis factoriales confirmatorios y mediante la validez convergente y divergente de ambos cuestionarios. La validez de contenido se investigó a través del criterio de jueces expertos, mientras que la validez predictiva se llevó a cabo a través de correlaciones y regresiones entre las variables estudiadas (clima y regulación del aprendizaje) y el rendimiento académico de los alumnos. La confiabilidad se verificó a través del método de consistencia interna (Alfa de Cronbach).

motivación / teoría de la autodeterminación / clima de aprendizaje / autonomía / control / autorregulación del aprendizaje

\section{Adaptation of two questionnaires of motivation: Learning Self-regulation Questionnaire and the Learning Climate Questionnaire}

This psychometric research aimed at adapting and validating two questionnaires: the Learning Self-regulation Questionnaire and the Learning Climate Questionnaire. The sample included 369 university students from a private university in Lima which had a mean age of $19.30(S D=2.49)$. For both questionnaires, the construct validity was analyzed by principal components and by confirmatory factor analyses as well as convergent and divergent validity. Content analysis was evaluated by a panel of expert judges while predictive validity was analyzed by correlation and regression analyses between the studied variables (learning climate and learning self-regulation) and students' academic achievement. Reliability was evaluated by the internal consistency coefficient (Cronbach Alpha).

motivation / self-determination / learning climate / autonomy / control learning self-regulation

Correo electrónico: 1matos@ulima.edu.pe 


\section{INTRODUCCIÓN}

Uno de los constructos más interesantes en psicología es la motivación. Esta se refiere a la energía, dirección y persistencia del comportamiento (Ryan \& Deci, 2000). Pero, ¿por qué este constructo es tan importante en la vida? La respuesta tiene que ver con las consecuencias de la motivación, pues "la motivación produce" (Ryan \& Deci, 2000, p. 69).

La presente investigación psicométrica busca adaptar y validar dos instrumentos psicológicos para el estudio de la motivación en estudiantes universitarios. Es importante resaltar que la mayor parte de los cuestionarios disponibles para el estudio de distintos constructos psicológicos han sido desarrollados en otro idioma, básicamente en inglés. Por este motivo se requiere realizar la traducción y adaptación de estos instrumentos a nuestra realidad de acuerdo con las normas de la Comisión Internacional de Test (ITC, 2000). Esta comisión tiene como uno de sus lineamientos principales considerar las posibles diferencias lingüísticas y culturales existentes entre la población para la cual ha sido diseñada una prueba y aquella población en la que se pretende utilizar el instrumento traducido y adaptado. La idea es que se logre "capturar" la esencia de lo que se quiere evaluar, respetando los contextos culturales específicos.

Se estudió el constructo psicológico de motivación de acuerdo con la Teoría de la Autodeterminación (Self-determination Theory, SDT; Deci \& Ryan, 2002), debido a que es una de las más empleadas actualmente en el estudio de la motivación (Deci \& Ryan, 2002). Esta es una de las teorías más exhaustivas y con gran soporte empírico. Se centra en el grado en que la conducta humana es autodeterminada, es decir, en el grado en que las personas llevan a cabo sus acciones con un sentido de autonomía. Además, esta teoría tiene gran aplicación en diferentes ámbitos, especialmente en los contextos deportivo, clínico, de salud y educativo, entre otros (Deci \& Ryan, 2002).

Como se sabe, los estudiantes necesitan estar motivados para participar de manera activa en el proceso de enseñanza-aprendizaje y para continuar aprendiendo durante toda su vida (Boekaerts, 1996; Phalet \& Lens, 1995). La motivación puede tener influencia en sobre qué, cuándo y cómo aprendemos (Schunk, 1995). Los estudiantes motivados para aprender están dispuestos a comprometerse en actividades en las que ellos creen que van a aprender, tales como: escuchar atentamente las clases, organizar la información y repasar el material por aprender, verificar su nivel de comprensión del material que se estudiará, y pedir ayuda cuando no entienden lo que estén estudiando (Zimmerman, 2000). De tal modo, estas actividades en conjunto favorecen el aprendizaje (Schunk, Pintrich \& Meece, 2008). 
Todo sistema educacional exitoso exige estudiantes motivados, pero también es importante señalar que los estudiantes motivados requieren de profesores motivados, ya que de ellos depende crear un ambiente adecuado para el aprendizaje (Boekaerts, 1996).

Uno de los aspectos centrales de la teoría de la autodeterminación se refiere a la influencia que tiene la calidad de los contextos sociales en la motivación, el rendimiento y el bienestar de las personas que se encuentran inmersas en ese contexto social. Debido a esto, se ha incluido en el presente estudio el Cuestionario de Clima de Aprendizaje (véase el anexo 1). Este cuestionario permite evaluar si los contextos sociales, tales como los que se pueden dar en un salón de clases, en un equipo de fútbol o en una empresa, promueven o apoyan la autonomía personal (la autonomía es una de las necesidades básicas de las personas). Se ha encontrado que los contextos que apoyan la autonomía (versus el control) tienden a facilitar una motivación autodeterminada, un desarrollo saludable y un funcionamiento óptimo (Reeve, 2002).

La Teoría de la Autodeterminación (Deci \& Ryan, 2002) también distingue entre diferentes tipos de regulación del comportamiento, esto es, el grado en que estos tipos representan un funcionamiento autónomo o autodeterminado versus un comportamiento controlado. Así, la motivación intrínseca es el mejor ejemplo del comportamiento autó- nomo y, por lo tanto, autodeterminado. De otro lado, la motivación extrínseca es más controlada (y, por ende, menos autónoma). Existen cuatro tipos de regulación del comportamiento, que hacen referencia al grado en que la regulación de una actividad extrínsecamente motivada ha sido internalizada e integrada. En concordancia con ello, existen cuatro tipos de regulación: externa, introyectada (regulación de la conducta que no se acepta como propia), identificada (aceptación del valor de la actividad como personalmente importante) e integrada (además de aceptar el valor de la actividad como importante, se le acepta como parte de uno). Las regulaciones externas e introyectadas son catalogadas como formas controladas de motivación extrínseca, mientras que a las regulaciones identificadas e integradas se las considera relativamente autónomas. Dos tipos de regulación, esto es, autónoma y control, son evaluadas por el Cuestionario de Autorregulación del Aprendizaje (véase el anexo 2), que estudia las razones por las cuales las personas aprenden en ambientes académicos específicos, como colegios y universidades, entre otros (Deci \& Ryan, 2002).

Tomando en cuenta la importancia que la motivación tiene sobre el comportamiento de los estudiantes, resulta necesario contar con instrumentos válidos y confiables en el Perú, para poder examinar cómo la motivación de los alumnos y los contextos educativos fa- 
vorecen el aprendizaje y el rendimiento académico.

A continuación se plantean los objetivos de la presente investigación.

\section{Objetivos}

Los objetivos del presente proyecto de investigación son:

- Objetivo general

- Traducir y adaptar dos instrumentos de evaluación de la motivación a una población universitaria.

- Objetivos específicos

- Traducir los dos instrumentos de evaluación de la motivación siguiendo las normas de la Comisión Internacional de los Test (ITC, 2000).

- Estudiar las propiedades psicométricas (validez y confiabilidad) de los dos instrumentos en nuestro medio.

\section{MÉTOdo}

\section{Participantes}

Los participantes de esta investigación fueron en total 369 estudiantes universitarios, de los cuales $138(37,4 \%)$ eran hombres y $228(61,8 \%)$ eran mujeres (3 participantes no reportaron su género). Los alumnos tenían una edad promedio de 19.30 años $(D S=2.49)$, y sus edades estuvieron en un rango de 16 a
32 años de edad. La edad promedio tanto de los hombres $(D S=3)$ como de las mujeres $(D S=2)$ fue de 19 años.

La mayoría de participantes procedían de los dos primeros ciclos $(N=202,54,7 \%)$, si bien también fueron tomados en cuenta otros (desde el primero al décimo ciclo). Un total de 336 participantes (91\%) perteneció a colegios privados, mientras que el resto $(N=33,9 \%)$ provenía de colegios estatales. La mayoría de participantes nació en la ciudad de Lima $(N=303$, $82,1 \%$ ) y una pequeña parte de la muestra era de provincias $(N=57$, $15,4 \%$ ), o había nacido en el extranjero $(N=6,1,6 \%)$, aunque algunos prefirieron no mencionar su lugar de nacimiento $(N=3,0,8 \%)$. La mayoría de participantes reportaron vivir en los distritos de Surco $(N=96,26 \%)$, La Molina $(N=47,12,7 \%)$ y San Borja $(N=32,8,7 \%)$.

Los alumnos evaluados provenían de diversas facultades. La facultad con la mayor cantidad de alumnos fue la de Psicología, con un total de 154 (42\%) participantes, seguida por las facultades de Ingeniería $(N=48,13 \%)$ y Administración $(N=37,10 \%)$.

La mayoría de participantes reportaron que sus padres tuvieron una educación superior. Con relación a la madre, superior técnica $(N=115,31,2 \%)$, o superior universitaria $(N=136$, $36,9 \%$ ), y con relación al padre, superior técnica $(N=49,13,3 \%)$, o superior universitaria $(N=225,61 \%)$. Fueron 
pocos los alumnos que declararon que sus padres no terminaron la educación escolar (primaria o secundaria), ya sea con respecto a la madre $(N=7,1,9 \%)$ o al padre $(N=24,6,5 \%)$. Las ocupaciones más comunes de las madres de familia fueron: profesora $(N=38$, $10,3 \%)$, secretaria $(N=25,6,8 \%)$, contadora $(\mathrm{N}=21,5,7 \%)$ o administradora $(\mathrm{N}=21,5,7 \%)$. $\mathrm{Y}$ en el caso de los padres de familia: ingeniero $(N=67$, $18,2 \%)$, administrador $(N=40,10,8 \%)$ y contador $(N=23,6,2 \%)$.

\section{Instrumentos}

\section{Cuestionario de Clima de Aprendizaje}

El Cuestionario de Clima de Aprendizaje (Williams \& Deci, 1996) consta de 15 ítems, los cuales deben ser respondidos en una escala Likert que va del 1 (Totalmente en desacuerdo) a 7 (Totalmente de acuerdo). En este cuestionario se evalúa (en una sola escala) la percepción de los participantes acerca del grado en que el líder (por ejemplo, el profesor, el entrenador, etcétera) promueve la autonomía de sus alumnos. Así, los puntajes más altos en esta escala indican un mayor apoyo a la autonomía de los alumnos por parte del profesor.

El Cuestionario de Clima de Aprendizaje ha obtenido buenas propiedades psicométricas en estudiantes universitarios. La validez factorial de la escala fue investigada inicialmente por $\mathrm{Wi}$ - 1liams \& Deci (1996), quienes reportaron que los 15 ítems del cuestionario forman una sola escala, ya que se obtuvo un autovalor (Eigen) de 9.5 que representó el $63 \%$ de varianza explicada. Siguiendo con la validez de constructo, dichos autores reportaron buena validez convergente y divergente, debido a que el puntaje total del Cuestionario de Clima de Aprendizaje correlacionó positivamente $(r=.24, p<.05)$ con la subescala de Percepción de la Autonomía de la "Escala General de Orientación de la Causalidad" (GCOS, Deci \& Ryan, 1985), y no obtuvo correlaciones significativas con la subescala de Control o Impersonal de la misma prueba. Esta escala ha reportado buena consistencia interna, puesto que se han logrado índices alfa de Cronbach de 0.93 y 0.94 (Black \& Deci, 2000) y 0.96 (Williams \& Deci, 1996; Williams, Saizow, Ross \& Deci, 1997).

\section{Cuestionario de Autorregulación del} Aprendizaje

El Cuestionario de Autorregulación del Aprendizaje consta de 14 ítems, en los cuales los participantes deben responder qué tan verdadero o qué tan falso es cada una de las frases, de acuerdo con una escala Likert que va del 1 (Para nada verdadero) al 7 (Totalmente verdadero).

El referido cuestionario somete a evaluación el grado de autorregulación 
del aprendizaje en dos escalas: Autonomía y Control. Ambas evalúan dos formas diferentes de autorregulación: por un lado, la autonomía en el aprendizaje, que se define como el grado en que la actividad de aprender es realmente importante para la persona (regulación interna). Por otro lado, se evalúa el control, el cual es conceptuado como el grado en que la persona regula su aprendizaje de manera externa, es decir, se regula la conducta a partir de elementos que no son propios (ejemplo: hacer lo que dice el profesor, por temor a la represalia o al castigo, etcétera).

El Cuestionario de Autorregulación del Aprendizaje ha obtenido buenas propiedades psicométricas en estudiantes universitarios. Con relación a la validez factorial, se reportó que se lograron autovalores (Eigen) de 4.7 para la escala de Autonomía y 2.6 para la escala de Control. No se informó, sin embargo, acerca de cuánta varianza explicaron estos valores (Williams \& Deci, 1996).

Con relación a la validez de constructo del Cuestionario de Autorregulación del Aprendizaje, la subescala de Autonomía correlacionó positivamente $(r=.33, p<.001)$ con la subescala de Autonomía de la "Escala General de Orientación de la Causalidad" (GCOS, Deci \& Ryan, 1985), y la escala de Control correlacionó también positivamente con las subescalas de Control $(r=.27, p<.001)$ e Impersonal $(r=.45, p<.001)$ de la GCOS, lo que demuestra la validez convergente y divergente de la escala. Asimismo, ha sido reportada la consistencia interna de este cuestionario, habiéndose obtenido índices de 0.78 y 0.70 para las escalas de Autonomía y de Control, respectivamente (Williams \& Deci, 1996).

\section{Procedimiento}

Como primer paso, se hizo la traducción de los instrumentos del inglés al castellano. Luego, un investigador experto realizó la traducción inversa (back-translation) para poder comparar las dos versiones, método empleado frecuentemente para evaluar la precisión de la traducción.

Si bien es difícil que las palabras específicas entre la versión traducida y la original coincidan exactamente, se aceptan mientras se mantenga el contenido del mensaje de manera clara. Se procedió posteriormente a comparar la versión original y la versión de la traducción inversa, y se hicieron las correcciones y cambios convenientes para asegurar que la nueva versión en castellano mantuviera el sentido y contenido de los ítems de la versión original de la prueba. Luego, esta versión en español fue sometida a un grupo de jueces $(N=7)$; en este caso, psicólogos que dominaban el idioma inglés, recogiéndose sus sugerencias y efectuando las modificaciones necesarias. 
Para saber si esta versión era comprendida totalmente, se realizó un estudio piloto $(N=20)$ con un grupo de estudiantes. Ellos respondieron los cuestionarios y, posteriormente, se les preguntó qué tan bien comprendían los ítems que conforman la prueba y sobre las dificultades que encontraron al responderla. Se recogieron sus comentarios y sugerencias y se pasó, finalmente, a la aplicación de los instrumentos a los diferentes grupos de la muestra.

Para recolectar la información, se contactó a los profesores y se les pidió su colaboración con la presente investigación. Luego se les entregó el sobre con los cuestionarios y procedieron a realizar las aplicaciones en sus respectivos salones. Los alumnos respondieron los cuestionarios en un tiempo que osciló entre 20 y 30 minutos.

\section{Plan de ANálisis estadísticos}

Para analizar las propiedades psicométricas de los cuestionarios se evaluaron: 1) la validez de contenido, la validez de constructo (validez factorial, convergente y divergente) y la validez predictiva de las pruebas, y 2) la confiabilidad, a partir de la evaluación de la consistencia interna de ambas pruebas.

\section{Resultados}

Los resultados de la investigación se ofrecen en dos partes: la primera presenta los resultados del proceso de tra- ducción y adaptación de los instrumentos, y la segunda los análisis de sus propiedades psicométricas.

\section{Traducción de la prueba}

Cada uno de los instrumentos fue traducido del inglés al español por un experto. Dentro del proceso de traducción de los cuestionarios se estudió también la validez de contenido, ya que los jueces no solamente evaluaron la idoneidad de la traducción y la adaptación, sino que, con ellas, se evaluó también el contenido del ítem y su referencia con el constructo teórico evaluado.

Como se mencionó en la parte concerniente a los instrumentos, un investigador experto realizó la traducción inversa (back-translation) para revisar la precisión y la pertinencia de la traducción. Se cuidó que el contenido de cada ítem en inglés se viera reflejado en la traducción al castellano. Se compararon ambas versiones y se hicieron correcciones convenientes para asegurar que la nueva versión en castellano mantenga el sentido de los ítems de la versión original de la prueba.

Con respecto al Cuestionario de Clima de Aprendizaje, el índice de acuerdo entre los jueces con respecto a la pertinencia de la traducción fue de 0.81 , mientras que para el Cuestionario de Autorregulación del aprendizaje fue de .84, siendo ambos índices altos. 
RESULTADOS DE LOS ANÁLISIS PSICOMÉTRICOS DE LOS CUESTIONARIOS

\section{Validez de contenido}

La validez de contenido evalúa la medida en que la prueba representa todas las facetas del constructo teórico que es evaluado. El proceso de traducción de los cuestionarios se utiliza también como un proceso para comprobar la validez de contenido de los instrumentos, ya que los jueces no solamente evalúan la idoneidad de la traducción, sino que también se les pidió que evalúen el contenido del ítem y su relación con los constructos teóricos que se están estudiando. Es decir, los jueces dictaminan si el ítem (ya traducido) permite evaluar lo que se supone se debe evaluar en cada instrumento (incluyendo sus subescalas). En relación con el contenido de los ítems, se observó que con respecto al Cuestionario de Clima de Aprendizaje, el índice de acuerdo entre los jueces fue de 0.89 , mientras que para el Cuestionario de Autorregulación del aprendizaje fue de .90 , siendo ambos índices altos. Por lo tanto, podemos decir que los cuestionarios cuentan con validez de contenido.

\section{Validez de constructo}

Con el propósito de analizar la validez de constructo de los instrumentos, se realizó inicialmente un análisis factorial exploratorio (AFE, con extracción de componentes principales), en el que se analizaron los autovalores (Eigen), el gráfico de sedimentación de Cattell (Scree Plot) y las cargas factoriales para determinar los ítems que forman parte de cada componente. Idealmente, las cargas factoriales en los análisis exploratorios deben ser iguales o mayores a .40 (Field, 2005).

Seguidamente se llevó a cabo un análisis factorial confirmatorio (AFC, Joreskog \& Sörbom, 2001). Los resultados del AFC fueron evaluados mediante la consideración de diversos índices de ajuste: 1) la proporción del Chi cuadrado (que se calcula dividiendo el Chi cuadrado entre los grados de libertad), para lo cual es necesario tener información acerca del chi cuadrado $\left(\chi^{2}\right)$ y los grados de libertad (gl); cuando los datos no estaban distribuidos normalmente, se empleó el Satorra-Bentler chi cuadrado (S-B $\chi^{2}$ ); 2) el RMSEA (raíz cuadrada media de error de aproximación); y 3) el CFI (índice de ajuste comparativo). Valores de la proporción del Chi cuadrado menores a 3 indican una buena adecuación del modelo (Kline, 1998). Valores cercanos a 0.08 en el RMSEA (Byrne, 1998) y valores superiores a 0.90 en el CFI muestran una buena adecuación del modelo $(\mathrm{Hu} \&$ Bentler, 1999; Kline, 1998). Asimismo, se analizaron las cargas factoriales, las cuales debían ser significativas $(p<.05)$.

El análisis de la validez de constructo continuó con la evaluación de la validez convergente y divergente de los 
cuestionarios. La validez convergente se evaluó realizando una correlación con otro instrumento ya existente que medía el mismo constructo, o con un instrumento que evalúe un constructo con el que debe tener una relación positiva teóricamente. En el caso de los cuestionarios por evaluarse, el Cuestionario de Clima de Aprendizaje (de apoyo a la autonomía) debería correlacionar positivamente con la escala de Autonomía del Cuestionario de Autorregulación del Aprendizaje para poder comprobar la validez convergente de ambas escalas.

Para evaluar la validez divergente, la correlación entre las escalas que evalúan constructos teóricos diferentes debe ser negativa o no significativa. En este caso, el Cuestionario de Clima de Aprendizaje y la escala de Autonomía del Cuestionario de Autorregulación del Aprendizaje tendrían que correlacionar negativamente (o de manera no significativa) con la escala de Control del Cuestionario de Autorregulación del Aprendizaje.

A continuación se reportan los resultados de los análisis realizados para analizar la validez de constructo de ambas pruebas.

\section{Validez factorial del Cuestionario de Clima de Aprendizaje}

Se inició la inspección de la validez de constructo mediante un análisis factorial exploratorio (AFE) con extracción de componentes principales con rotación Varimax. El resultado de estos análisis mostró que la medida de adecuación de la muestra Kayse-Meyer-Olkin (KMO) fue de .94, y el test de esfericidad de Bartlett fue altamente significativo $(p<.001)$, por lo que se pudo continuar con la interpretación de los resultados del análisis. Los resultados del test mostraron una sola escala con un autovalor (Eigen) de 7.72, el cual explicaba el $51,48 \%$ de la varianza. Luego de inspeccionar el gráfico de sedimentación de Cattell ("Scree Plot", véase la figura 1), se observó que, efectivamente, los valores están concentrados alrededor de un solo factor. Las cargas factoriales de los ítems alcanzaron valores entre 0.64 y 0.81 , con excepción del ítem 13, que alcanzó una carga factorial de 0.30 (véase la tabla 1). Se decidió mantener el ítem 13, puesto que la carga factorial, si bien es menor a 0.40 , resulta aceptable, y así se respeta la totalidad de la escala original.

Para corroborar la estructura factorial de la escala se realizó un análisis factorial confirmatorio (AFC) utilizando el programa Lisrel versión 8.5 (Jöreskog \& Sörbom, 2001). En este análisis se propuso reproducir los resultados del análisis exploratorio. Como ya fue mencionado, los índices de bondad de ajuste evaluados en el AFC fueron: el Satorra-Bentler chi cuadrado $\left(\mathrm{S}-\mathrm{B} \chi^{2}\right)$, la razón del chi cuadrado (S-B $\left.\chi^{2} / g 1\right)$, el RMSEA y el CFI. Los resultados mostraron logros satisfacto- 
Figura 1

Gráfico de sedimentación de Cattell ("Scree Plot”) del Cuestionario de Clima de Aprendizaje

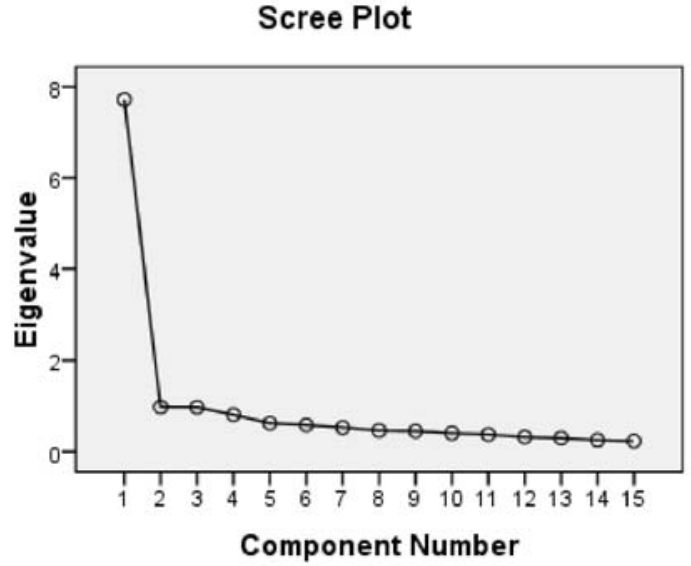

Tabla 1

Cargas factoriales del análisis de componentes principales y del análisis factorial confirmatorio (AFC)

\begin{tabular}{|c|c|c|}
\hline Ítem & Componentes principales & AFC \\
\hline 1 & .64 & $.31^{\star \star \star}$ \\
\hline 2 & .77 & $.74^{\star \star \star}$ \\
\hline 3 & .71 & $.68^{\star * \star}$ \\
\hline 4 & .78 & $.76^{* * *}$ \\
\hline 5 & .78 & $.76^{\star * *}$ \\
\hline 6 & .70 & $.67^{* * *}$ \\
\hline 7 & .66 & $.64^{\star * *}$ \\
\hline 8 & .82 & $.81^{* * *}$ \\
\hline 9 & .78 & $.76^{\star \star \star}$ \\
\hline 10 & .76 & $.74^{\star * \star}$ \\
\hline 11 & .77 & $.75^{\star \star \star}$ \\
\hline 12 & .80 & $.79^{\star * *}$ \\
\hline 13 & .30 & $.31^{* *}$ \\
\hline 14 & .68 & $.64^{\star \star *}$ \\
\hline 15 & .66 & $.62^{* \star *}$ \\
\hline
\end{tabular}

Nota.- ${ }^{* *} p<.01,{ }^{* * *} p<.001, N=369$ 
rios: $\mathrm{S}-\mathrm{B} \chi^{2} / \mathrm{gl}=2.17\left(\mathrm{~S}-\mathrm{B} \chi^{2}=195.49\right.$, $\mathrm{gl}=90), \mathrm{RMSEA}=0.058, \mathrm{CFI}=0.99$. Las cargas factoriales de los ítems alcanzaron valores entre 0.25 y 0.81 , siendo todas significativas (véase la tabla 1).

Estos resultados son similares a los del análisis factorial exploratorio e indican que el cuestionario posee una sola escala. Las cargas factoriales de los análisis factoriales exploratorio y confirmatorio pueden verse en la tabla 1 .

\section{Validez factorial del Cuestionario de Autorregulación del Aprendizaje}

Se realizó la validez de constructo mediante el AFE y se utilizó un análisis de componentes principales con rotación Varimax. El resultado del análisis mostró que la medida de adecuación de la muestra Kayser-Meyer-Olkin (KMO) fue de .76, y el test de esfericidad de Bartlett fue altamente significativo $(p<.001)$, por lo que se pudo continuar con la interpretación de los resultados del análisis.

El resultado del análisis de componentes principales resultó con tres componentes con autovalores (Eigen) mayores a 1 , los cuales explicaron un $58,9 \%$ del total de la varianza.

El autovalor (Eigen) del primer componente fue de 3.4 y explicó el $24,25 \%$ de la varianza. El segundo componente obtuvo un autovalor de 2.85 explicando el $20,38 \%$ de la varianza, mientras que el tercer compo- nente obtuvo un autovalor de 2 , y explicó un $14,29 \%$ del total de la varianza.

Las cargas factoriales fueron desde 0.10 hasta 0.76 , y varios de los ítems compartían cargas factoriales altas en diversos componentes. Dados los resultados de este análisis inicial y la necesidad de probar un modelo de dos escalas, se realizó un nuevo AFE donde se calculó las cargas factoriales para dos componentes. El resultado del análisis mostró que la medida de adecuación de la muestra Kayser-Meyer-Olkin (KMO) fue de .76, y el test de esfericidad de Bartlett fue altamente significativo $(p<.001)$, por lo que se pudo continuar con la interpretación de los resultados del análisis.

Este análisis resultó en dos componentes con autovalores (Eigen) de 3.42 y 3.37 explicando el $24,4 \%$ y el $24 \%$ de la varianza total, respectivamente. Ambos componentes explicaron el 48,4\% del total de la varianza. Esta vez, los ítems alcanzaron cargas factoriales con valores entre 0.39 y 0.86 (véase la tabla 2). Sin embargo, algunos ítems contenían cargas factoriales altas en los dos componentes, y ello no permitió realizar un juicio definitivo acerca de la estructura factorial de la escala mediante estos análisis. Debe tenerse en cuenta, como ya se indicó, que dichos análisis eran solo de carácter exploratorio.

Finalmente, para establecer si la estructura factorial del cuestionario puede ser reproducida en esta muestra de par- 
ticipantes, se realizó el Análisis Factorial Confirmatorio. Como resultado de este análisis se obtuvieron los siguientes índices de ajuste: $\mathrm{S}-\mathrm{B} \chi^{2} / \mathrm{gl}=2.25$ $\left(\mathrm{S}-\mathrm{B} \chi^{2}=157.22, \mathrm{gl}=70\right), \mathrm{RMSEA}=$ 0.077 , CFI $=0.90$, lo que demuestra que la estructura factorial planteada originalmente por el cuestionario se reproduce aceptablemente en la presente muestra. Las cargas factoriales alcanzaron valores entre 0.26 y 0.86 y todas fueron significativas (véase la tabla 2). Así, los resultados del AFC demuestran la validez de constructo del Cuestionario de Autorregulación del Aprendizaje. Las cargas factoriales de los análisis factoriales exploratorio y confirmatorio pueden verse en la tabla 2 .

\section{Validez convergente y divergente de los cuestionarios}

Para evaluar la validez convergente y divergente de las pruebas se realizó una correlación entre los dos cuestionarios, específicamente entre el Cuestionario del Clima de Aprendizaje y las escalas de Autonomía y Control del Cuestionario de Autorregulación del aprendizaje. Los resultados de estos análisis pueden verse en la tabla 3.

Como puede observarse en ella, el Cuestionario del Clima de Aprendizaje correlacionó positivamente $(r=.49$, $p<.001$ ) con la escala de Autonomía y no reportó una correlación significativa con la escala de Control $(r=.12$,

Tabla 2

Cargas factoriales del análisis de componentes principales y del análisis factorial confirmatorio (AFC) del Cuestionario de Autorregulación del Aprendizaje

\begin{tabular}{|c|c|c|c|c|}
\hline \multirow[t]{2}{*}{ Ítem } & \multicolumn{2}{|c|}{ AFE $(N=369)$} & \multicolumn{2}{|c|}{$\operatorname{AFC}(N=248)$} \\
\hline & Autonomía & Control & Autonomía & Control \\
\hline 1 & .54 & & $.33^{\star \star}$ & \\
\hline 2 & & .73 & & $.66^{\star \star *}$ \\
\hline 3 & .68 & & $.46^{\star \star \star}$ & \\
\hline 4 & & .47 & & $.43^{\star *}$ \\
\hline 5 & .31 & .54 & & $.26^{\star \star}$ \\
\hline 6 & .78 & & $.82^{\star \star *}$ & \\
\hline 7 & & .86 & & $.80^{\star * *}$ \\
\hline 8 & & .66 & & $.57^{\star \star}$ \\
\hline 9 & .81 & & $.86^{\star \star *}$ & \\
\hline 10 & & .76 & & $.70^{\star \star *}$ \\
\hline 11 & .77 & & $.69^{* * *}$ & \\
\hline 12 & .58 & & $.52^{\star \star \star}$ & \\
\hline 13 & .22 & .39 & & $.26^{* *}$ \\
\hline 14 & & .80 & & $.27^{* *}$ \\
\hline
\end{tabular}

Nota. Las cargas factoriales subrayadas indican su presencia $(>.20)$ en las dos escalas ${ }^{* *} p<.01,{ }^{* *} p<.001$. 
$p>.05)$. Por su parte, la escala de $\mathrm{Au}-$ tonomía solo correlacionó significativamente con el puntaje total del Cuestionario de Clima de Aprendizaje y no con la escala de Control de la Escala de Autorregulación del Aprendizaje $(r=.08, p>.05)$. Así, es posible observar con claridad que los cuestionarios estudiados poseen validez convergente y divergente.

Tabla 3

Análisis de correlación entre el Cuestionario del Clima de Aprendizaje (Clima) y las escalas de Autonomía y Control del Cuestionario de Autorregulación del Aprendizaje

\begin{tabular}{lcc}
\hline & Clima & Autonomía \\
\hline Autonomía & $.49^{\star * *}$ & \\
Control & .12 & .08 \\
\hline Nota.- $^{* * *} p<.001$ & &
\end{tabular}

Finalmente, se puede concluir que tanto el Cuestionario de Clima de Aprendizaje como el de Autorregulación del Aprendizaje poseen validez de constructo.

\section{Validez predictiva de los cuestionarios de Clima de Aprendizaje y de Autorregulación del Aprendizaje}

La validez predictiva de la escala se evaluó al investigar la relación entre los puntajes totales de los cuestionarios y el rendimiento académico de los alumnos en los cursos en los cuales fueron evaluados durante el semestre (ciclo 2008-
2). En la presente investigación, ello significa que el Cuestionario de Clima de Aprendizaje y la Escala de Autonomía del Cuestionario de Autorregulación del Aprendizaje deberían predecir positivamente el rendimiento académico de los alumnos, mientras que la escala de Control del Cuestionario de Autorregulación del Aprendizaje lo haría negativamente o no lo haría.

Para evaluar estas expectativas y para analizar qué variables son realmente predictoras del rendimiento académico se realizó primero un análisis de correlación entre las variables estudiadas. De esta manera, se exploraron las relaciones entre las variables.

Más adelante se presentaron dos análisis de regresión jerárquica múltiple (uno para cada cuestionario) con el fin de analizar qué variables fueron predictoras significativas del rendimiento académico de los alumnos.

Cada uno de los análisis de regresión múltiple se realizó en dos pasos: se analizó primero el rol de las variables relacionadas con la información demográfica recogida, las cuales fueron consideradas como variables de control, mientras que, en un segundo paso, se hizo lo propio con el rol de las variables correspondientes a los cuestionarios.

Los resultados mostraron que, de acuerdo con las expectativas, el Cuestionario de Clima de Aprendizaje (de apoyo a la autonomía) correlacionó po- 
sitivamente con el rendimiento académico de los alumnos, al igual que la escala de Autonomía del Cuestionario de Autorregulación del Aprendizaje. La escala de Control, por otro lado, no correlacionó significativamente con las notas de los alumnos (véase la tabla 4).

Tabla 4

Análisis de correlación entre el rendimiento académico (notas) y el

Clima de Aprendizaje (clima), Autonomía y Control.

\begin{tabular}{lccc}
\hline & Clima & Autonomía & Control \\
\hline Clima & & & \\
Autonomía & $.49^{\star *}$ & & \\
Control & .12 & .075 & \\
Notas & $.30^{\star *}$ & $.25^{\star *}$ & -.13 \\
\hline
\end{tabular}

Nota.- ${ }^{* \star} p<.01, N=148-241$

Los resultados de los análisis de regresión utilizados para conocer si las variables de estudio podían predecir el rendimiento académico de los alumnos pueden observarse en la tabla 5. Los análisis de regresión múltiple se realizaron por separado para cada cuestionario, ya que, como se aprecia en la tabla 4, la relación entre el clima de aprendizaje y la escala de autonomía es bastante alta, por lo que es posible que, al ser incluidos en un solo análisis de regresión, los posibles efectos individuales se omitan o se controlen.

Con relación al Cuestionario de Clima de Aprendizaje, el análisis de regresión múltiple mostró que las variables predictoras explicaron un total de $29 \%$ de la varianza del rendimiento académico. En el primer paso, las variables de Control (edad, ciclo de estudio, sexo, educación de la madre y educación del padre) explicaron el $18 \%$ de la varianza del rendimiento académico, siendo la variable Ciclo la única variable predictora significativa $(\beta=.37$, $p<.01)$ de las notas de los alumnos, lo cual indica que a mayor ciclo, mejores notas. En el segundo paso, el Clima de aprendizaje (de apoyo a la autonomía) explicó un $11 \%$ adicional de varianza en la variable Rendimiento académico $\mathrm{y}$ mostró ser un predictor significativo $(\beta=.34, p<.001)$ del rendimiento académico (véase la tabla 5). Esto quiere decir que mientras mayor sea el clima de aprendizaje de apoyo a la autonomía, mayor será el rendimiento académico de los alumnos.

Con relación al Cuestionario de Autorregulación del Aprendizaje, las variables predictoras explicaron un total de $22 \%$ de la varianza de la variable Rendimiento académico. En el primer paso, las variables de control explicaron el $17 \%$ de la varianza de la variable Rendimiento, siendo Ciclo la única variable predictora significativa $(\beta=.33$, $p<.01)$ de las notas de los alumnos. En el segundo paso, luego de añadir las variables Autonomía y Control en el Análisis, se pudo observar que estas explicaron un 5\% adicional de varianza en el rendimiento académico. La variable Autonomía mostró ser un predictor significativo $(\beta=.24, p<.01)$ del ren- 
dimiento académico (véase la tabla 5), y la variable Control no fue un predictor significativo de las notas de los alumnos $(\beta=-.11, p>.05)$. Sin embargo, puede observarse que el índice de Control es negativo. Los resultados demuestran que las expectativas planteadas se vieron confirmadas nuevamente.

Los resultados ponen de manifiesto, finalmente, que tanto el aumento en el clima de aprendizaje que promueve la autonomía, como el que se produce en la autorregulación del aprendizaje de manera autónoma, predicen positivamente el rendimiento académico de los alumnos, por lo que se puede inferir que ambos cuestionarios poseen validez predictiva.

\section{Análisis de la confiabilidad de los cuestionarios}

Luego de realizar los análisis de validez de los cuestionarios, queda solamente comprobar su confiabilidad. Estos dos cuestionarios ya han reportado buena confiabilidad en investigaciones anteriores.

Para analizarla se empleará el método de consistencia interna (Alfa de Cronbach), en el que se incluirá toda la muestra. Deberá revisarse, igualmente, la correlación de cada ítem con el total del factor, el cual debe ser igual o mayor a .30 (Field, 2005). El índice de consistencia interna (alfa de Cronbach) ha de ser de al menos .70 para que se le considere aceptable (Aiken, 1996).

Tabla 5

Coeficientes beta estandarizados $(\beta)$ de los análisis de regresión múltiples realizados para la predicción del rendimiento académico (Notas)

\begin{tabular}{|c|c|c|c|c|}
\hline & \multicolumn{2}{|c|}{$\begin{array}{l}\text { Cuestionario del Clima } \\
\text { de Aprendizaje }\end{array}$} & \multicolumn{2}{|c|}{$\begin{array}{c}\text { Cuestionario de Autorregulación } \\
\text { del Aprendizaje }\end{array}$} \\
\hline & $\begin{array}{c}\text { Paso } 1 \\
\left(R^{2}=.18\right)\end{array}$ & $\begin{array}{c}\text { Paso } 2 \\
\left(R^{2}=.11\right)\end{array}$ & $\begin{array}{l}\text { Paso } 1 \\
\left(R^{2}=.17\right)\end{array}$ & $\begin{array}{c}\text { Paso } 2 \\
\left(R^{2}=.05\right)\end{array}$ \\
\hline \multicolumn{5}{|l|}{ Variables de Control } \\
\hline Edad & .01 & .05 & .08 & .01 \\
\hline Ciclo & $.37^{\star *}$ & $.42^{* * *}$ & $.33^{* *}$ & $.32^{* *}$ \\
\hline Género & .10 & .13 & .09 & .10 \\
\hline Ed. de la madre & .06 & .02 & .06 & .02 \\
\hline Ed. del padre & -.02 & -.04 & -.04 & -.04 \\
\hline \multicolumn{5}{|l|}{ Cuestionarios } \\
\hline Clima & & $.34^{\star * *}$ & & \\
\hline Autonomía & & & & $.24^{\star *}$ \\
\hline Control & & & & -.11 \\
\hline
\end{tabular}

Nota.- ${ }^{* \star} p<.01,{ }^{* \star *} p<.01 ;$ Ed. = Educación, $N=148$ 
El índice de consistencia interna alfa de Cronbach para el Cuestionario de Clima de Aprendizaje fue de 0.93 y los índices de consistencia interna alfa de Cronbach para las escalas de Autonomía y Control del Cuestionario de Autorregulación del Aprendizaje fueron de 0.79 y 0.78 , respectivamente.

Los resultados muestran que la prueba alcanza buenos niveles de confiabilidad. Los cuestionarios adaptados en el estudio se incluyen en el anexo 1.

\section{CONCLUSIÓN}

En el presente trabajo se han analizado las propiedades psicométricas de dos cuestionarios: el de Clima de Aprendizaje (de apoyo a la autonomía) y el de Autorregulación del Aprendizaje. Luego de haber realizado los análisis de validez y confiabilidad, se ha encontrado que ambas pruebas poseen validez de contenido, validez de constructo y validez predictiva y, además, han demostrado ser confiables.

Con estos resultados se estaría contribuyendo con el desarrollo de un Banco de Pruebas de Evaluación de la Motivación Traducidas y Adaptadas a nuestra realidad, de tal modo que pueda ser empleada por estudiantes, profesores e investigadores de la comunidad científica universitaria que estén interesados en esta área, promoviéndose así en nuestro medio investigaciones de esta modalidad.

\section{REFERENCIAS}

Aiken, L. R. (1996). Tests psicológicos y evaluación. México: Prentice Hall Hispanoamericana.

Black, A. E. \& Deci, E. L. (2000). The effects of instructors' autonomy support and students' autonomous motivation on learning organic chemistry: A self-determination theory perspective. Science Education, 84, 740-756.

Boekaerts, M. (1996). Self-regulated learning at the junction of cognition and motivation. European Psychologist, 1(2), 100-112.

Comisión Internacional de Tests (ITC, 2000). Directrices Internacionales para el Uso de los Test. http:// www.intestcom.org/Downloads/IT C\%20Test\%20Use\%20Guidelines $\% 20-\% 20$ Spain.doc $>$.

Deci, E. L. \& Ryan, R. M. (1985). The general causality orientations scale: Self- determination in personality. Journal of Research in Personality, 19, 109-134.

Deci, E. L. \& Ryan, R. M. (Eds.) (2002). Handbook of self-determination research. Rochester, Nueva York: University of Rochester Press.

Field, A. (2005). Discovering statistics using SPSS for windows. Londres: Sage. 
Hu, L. \& Bentler, P. M. (1999). Cutoff criteria for fit indexes in covariance structure analysis: Conventional criteria versus new alternatives. Structural Equation Model ing, 6(1), 1-55.

Jöreskog, K. \& Sörbom, D. (2001). Lisrel 8: Structural equation modeling with the Simplis command language. Hillsdale, Nueva Jersey: Laurence Erlbaum.

Kline, Rex B. (1998). Principles and practice of structural equation modeling. Nueva York: Guilford Press.

Phalet, K. \& Lens, W. (1995). Achievement motivation and group loyalty among Turkish and Belgian youngsters. En P. R. Pintrich \& M. Maehr (Eds.). Advances in motivation and achievement, 9, (pp. 31-72). Greenwich: JAI Press Inc.

Reeve, J. (2002). Self-determination theory applied to educational settings. En E. L. Deci \& R. M. Ryan (Eds.). Handbook of self-determination research (pp. 183-203). Rochester, Nueva York: University of Rochester Press.

Ryan, R. M. \& Deci, E. L. (2000). SelfDetermination Theory and the Facilitation of Intrinsic Motivation,
Social Development, and Wellbeing. American Psychologist, 1, 66-78.

Schunk, D. (1995). Self-Regulation of Self-Efficacy and Attibutions in Academic Settings. En D. Schunk \& B. Zimmerman (Eds.). Self-regulation of learning and performance: Issues and educational applications (pp. 75-89). Nueva York, Hillsdale: Lawrence Erlbaum Associates.

Schunk, D. H.; Pintrich, P. R. \& Meece, J. L. (2008). Motivation in Education. Theory, Research, and Applications. (3. ${ }^{\mathrm{a}}$ ed). Upper Saddle River, Nueva Jersey: Pearson.

Williams, G. C. \& Deci, E. L. (1996). Internalization of biopsychosocial values by medical students: A test of self-determination theory. Journal of Personality and Social Psychology, 70, 767-779.

Williams, G. C.; Saizow, R.; Ross, L.; \& Deci, E. L. (1997). Motivation underlying career choice for internal medicine and surgery. Social Sci ence and Medicine, 45, 1705-1713.

Zimmerman, B. J. (2000). Attaining Self-Regulation: a social cognitive perspective. En M. Boekaerts; P. Pintrich \& M. Zeodmer (Eds.). Handbook of Self-Regulation. Academic Press. 


\section{ANEXO 1}

\section{Cuestionario de Clima de Aprendizaje}

Este cuestionario contiene una serie de frases que se refieren a tu relación con el profesor de la clase. Los profesores tienen diferentes estilos al tratar con los estudiantes, y deseamos saber un poco más cómo te has sentido en la relación con tu profesor. Tus respuestas son confidenciales. Por favor, sé honesto. Usa la siguiente escala para responder las siguientes afirmaciones:

1

Totalmente

en desacuerdo
23

Neutral

Neutral
6

Totalmente de acuerdo

1 Siento que mi profesor me da opciones y posibilidades de hacer elecciones.

2 Siento que mi profesor me comprende.

$\begin{array}{lllllll}1 & 2 & 3 & 4 & 6 & 6 & 7\end{array}$

$\begin{array}{lllllll}1 & 2 & 3 & 4 & 6 & 6 & 7\end{array}$

3 Durante la clase, tengo la posibilidad de "abrirme" (ser sincero) con este profesor.

$\begin{array}{lllllll}1 & 2 & 3 & 4 & 6 & 6 & 7\end{array}$

4 Mi profesor tiene confianza en mi habilidad y en que haré las cosas bien en esta clase.

5 Siento que mi profesor me acepta.

$\begin{array}{lllllll}1 & 2 & 3 & 4 & 6 & 6 & 7\end{array}$

$\begin{array}{lllllll}1 & 2 & 3 & 4 & 6 & 6 & 7\end{array}$

6 Mi profesor se asegura de que yo realmente haya entendido los objetivos del curso y lo que necesito hacer (en este curso).

7 Mi profesor me alienta a hacer preguntas.

8 Siento mucha confianza en mi profesor.

9 Mi profesor responde a todas mis preguntas cuidadosamente.

10 Mi profesor escucha cómo yo quisiera hacer las cosas.

11 Mi profesor maneja las emociones de las personas muy bien.

12 Siento que a mi profesor le importo como persona.

13 No me siento muy bien con respecto a la forma en que mi profesor me habla.

14 Mi profesor trata de entender cómo veo las cosas antes de sugerir nuevas maneras de hacerlas.

15 Me siento capaz de compartir mis emociones con mi profesor. $\begin{array}{lllllll}1 & 2 & 3 & 4 & 6 & 6 & 7\end{array}$

$\begin{array}{lllllll}1 & 2 & 3 & 4 & 6 & 6 & 7\end{array}$

$\begin{array}{lllllll}1 & 2 & 3 & 4 & 6 & 6 & 7\end{array}$

$\begin{array}{lllllll}1 & 2 & 3 & 4 & 6 & 6 & 7\end{array}$

$\begin{array}{lllllll}1 & 2 & 3 & 4 & 6 & 6 & 7\end{array}$

$\begin{array}{lllllll}1 & 2 & 3 & 4 & 6 & 6 & 7\end{array}$

$\begin{array}{lllllll}1 & 2 & 3 & 4 & 6 & 6 & 7\end{array}$

$\begin{array}{lllllll}1 & 2 & 3 & 4 & 6 & 6 & 7\end{array}$

$\begin{array}{lllllll}1 & 2 & 3 & 4 & 6 & 6 & 7\end{array}$

$\begin{array}{lllllll}1 & 2 & 3 & 4 & 6 & 6 & 7\end{array}$ 


\section{ANEXO 2}

\section{Cuestionario de Autorregulación del Aprendizaje}

Las siguientes afirmaciones se refieren a las razones que tienes para participar en las clases. Las personas tienen distintas razones para participar y queremos conocerlas. Por favor, usa la siguiente escala para responder a cada una de las siguientes frases. Tus respuestas son confidenciales.

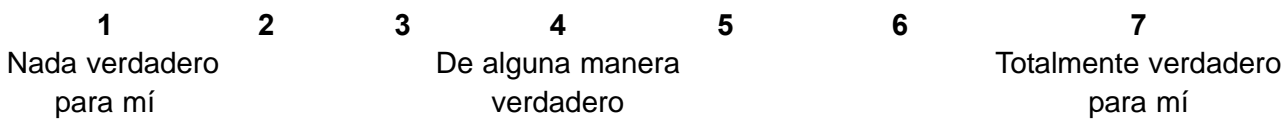

1 Yo participo activamente en las clases porque siento que es una buena manera de mejorar mis habilidades y la comprensión de los contenidos de los cursos.

2 Yo participo activamente en las clases porque otros pensarían mal de mí si no lo hiciera.

$\begin{array}{llllllll}1 & 2 & 3 & 4 & 5 & 6 & 7\end{array}$

$\begin{array}{llllllll}1 & 2 & 3 & 4 & 5 & 6 & 7\end{array}$

3 Yo participo activamente en las clases porque una comprensión profunda de mis clases es importante para mi crecimiento intelectual. $\begin{array}{llllllll}1 & 2 & 3 & 4 & 5 & 6 & 7\end{array}$

4 Yo participo activamente en las clases de mi carrera porque me sentiría mal conmigo mismo si es que no lo hago.

5 Yo sigo las sugerencias de mis profesores porque siguiéndolas, yo obtendré una buena nota.

6 Yo sigo las sugerencias de mis profesores porque pienso que me ayudarán a lograr un mejor aprendizaje.

7 Yo sigo las sugerencias de mis profesores porque quiero que otros piensen que soy bueno.

8 Yo sigo las sugerencias de mis profesores porque es más fácil hacer lo que me dicen que pensar acerca de eso.

9 Yo sigo las sugerencias de mis profesores porque es importante para mí aprender lo mejor que pueda.

10 Yo sigo las sugerencias de mis profesores porque probablemente me sentiría culpable si no lo hago.

11 La razón por la que continuaré ampliando mis conocimientos es porque es interesante aprender más.

12 La razón por la que continuaré ampliando mis conocimientos es porque es un reto comprender realmente lo que hacemos en los cursos.

13 La razón por la que continuaré ampliando mis conocimientos es porque las buenas notas en los cursos se verían muy bien en mi consolidado de notas.

14 La razón por la que continuaré ampliando mis conocimientos es porque quiero que otros vean que soy inteligente. $\begin{array}{lllllll}1 & 2 & 3 & 4 & 5 & 6 & 7\end{array}$

$\begin{array}{lllllll}1 & 2 & 3 & 4 & 5 & 6 & 7\end{array}$

$\begin{array}{lllllll}1 & 2 & 3 & 4 & 5 & 6 & 7\end{array}$

$\begin{array}{lllllll}1 & 2 & 3 & 4 & 5 & 6 & 7\end{array}$

$\begin{array}{lllllll}1 & 2 & 3 & 4 & 5 & 6 & 7\end{array}$

$\begin{array}{lllllll}1 & 2 & 3 & 4 & 5 & 6 & 7\end{array}$

$\begin{array}{lllllll}1 & 2 & 3 & 4 & 5 & 6 & 7\end{array}$

$\begin{array}{lllllll}1 & 2 & 3 & 4 & 5 & 6 & 7\end{array}$

$\begin{array}{lllllll}1 & 2 & 3 & 4 & 5 & 6 & 7\end{array}$

$\begin{array}{lllllll}1 & 2 & 3 & 4 & 5 & 6 & 7\end{array}$

$\begin{array}{lllllll}1 & 2 & 3 & 4 & 5 & 6 & 7\end{array}$ 